\title{
Arteriosclerotic Cardiovascular Disease
}

National Cancer Institute

\section{Source}

National Cancer Institute. Arteriosclerotic Cardiovascular Disease. NCI Thesaurus. Code C34403.

Cardiovascular disease resulting from arteriosclerosis. 